\title{
Memantine Treatment in Patients with Mild to Moderate Alzheimer's Disease: Results of a Randomised, Double-Blind, Placebo-Controlled 6-Month Study
}

\author{
S. Bakchine ${ }^{\mathrm{a}, *}$ and $\mathrm{H}$. Loft ${ }^{\mathrm{b}}$ \\ ${ }^{a}$ Hospital Maison Blanche, Department of Neurology, Reims University Hospital, Reims, France, INSERM EA 3797 \\ ${ }^{\mathrm{b}} H$. Lundbeck A/S, Copenhagen, Denmark
}

\begin{abstract}
Memantine is a moderate affinity, uncompetitive NMDA receptor antagonist currently approved for the treatment of moderate to severe Alzheimer's disease (AD). A 24-week, double-blind, placebo-controlled, study (Study 99679) conducted in Europe evaluated the efficacy and tolerability of $20 \mathrm{mg} /$ day memantine in patients with mild to moderate AD. Patients were randomised to either memantine or placebo in a 2:1 ratio. Efficacy was primarily assessed as change from baseline in ADAS-cog and CIBIC-plus score. Of 470 patients randomised and treated (memantine, $n=318$; placebo, $n=152$ ), $85 \%$ and $91 \%$ completed the study. Memantine-treated patients showed statistically significant improvement relative to placebo at weeks 12 and 18 , and numerical superiority at week 24 on both efficacy scales. The lack of significance at week 24 was attributed to an unexpectedly high placebo response. Memantine was well tolerated with an adverse event profile similar to placebo. The data presented support the efficacy of memantine in mild to moderate AD.
\end{abstract}

Keywords: Memantine, Alzheimer's disease, mild to moderate, NMDA receptor

\section{INTRODUCTION}

Alzheimer's disease (AD) is a chronic illness with progressive neurodegeneration and dementia. It has been estimated that $\mathrm{AD}$ affects approximately $1 \%$ of the population aged between 65 and 69 years and up to $20 \%$ of those aged above 85 years $[8,11,14]$. Clinically, AD is characterised by progressive decline in cognitive as well as functional capacities and a corresponding increase in the burden on caregivers. Pathological features of AD include extracellular amyloid

${ }^{*}$ Corresponding author: Serge Bakchine, Department of Neurology, Hospital Maison Blanche, 45 rue Cognacq Jay, 51092 REIMS, France. Tel./Fax: +33 3267871 35/+33 3267843 19; E-mail: sbakchine@chu-reims.fr. plaques containing the amyloid- $\beta$ protein and intraneuronal neurofibrillary tangles consisting of hyperphosphorylated tau protein, leading to loss of synapses as well as neuronal cell death. In addition to these structural changes, there are anatomic as well as functional changes in a number of neurotransmitter systems associated with cognition, including the glutamatergic and cholinergic pathways [3,32].

At present, the pharmacotherapy of mild to moderate $\mathrm{AD}$ is dominated by acetylcholinesterase inhibitors (AChEIs), which enhance central cholinergic neurotransmission. Although AChEIs have modest but measurable benefits, a number of patients have tolerability problems or contra-indications to members of this class of drugs. Given the multiple deficits in neurotransmission observed in $\mathrm{AD}$, no pharmacological agent tar- 
geting a single biochemical pathway can reasonably pretend to provide a major symptomatic improvement. Thus, there is a strong need for additional pharmacological treatment options in the management of the early stages of $\mathrm{AD}$. One potentially useful agent in this context is memantine. Memantine is the first drug in a new class of dementia therapies that act on the glutamatergic system via antagonism of the N-methyl-Daspartate (NMDA) receptor. Memantine has been approved for the treatment of moderate to severe $A D$ in both Europe and the US.

Memantine is a moderate affinity, uncompetitive NMDA receptor antagonist with strong voltagedependency and rapid blocking/unblocking kinetics [18]. These pharmacological features allow memantine to block the pathological sustained activation of the receptor hypothesised to occur in $\mathrm{AD}$, while rapidly leaving the NMDA receptor channel during normal physiological activation, for example, during learning and memory formation [12,18,27,38]. Placebocontrolled studies have demonstrated efficacy and safety of memantine monotherapy in moderate to severe $\mathrm{AD}$ outpatients as well as in severely demented nursing home patients $[25,26,37]$. In clinical studies in patients receiving ongoing treatment with an AChEI, memantine/AChEI has been shown to be superior to placebo/AChEI in the treatment of moderate to severe $\mathrm{AD}$ [33], as well as showing some benefits in patients with mild to moderate $\mathrm{AD}$ [Porsteinsson et al., in press]. Recently published monotherapy study in mild to moderate $\mathrm{AD}$ reported significant benefits of memantine also in these patients [20]. Furthermore, two multicentre, placebo-controlled studies in mild to moderate vascular dementia provide evidence that memantine may ameliorate cognitive symptoms in earlier stages of dementia $[17,35]$. In the light of these findings, the present study sought to investigate the efficacy and safety of memantine in patients with mild to moderate AD.

\section{MATERIALS AND METHODS}

\section{Study design}

This randomised, double-blind, parallel-group, placebo controlled, fixed-dose study of memantine in patients with mild to moderate $\mathrm{AD}$ included 65 primary care centres in 12 countries (Austria, Belgium, Denmark, Finland, France, Greece, Lithuania, the Netherlands, Poland, Spain, Sweden and United Kingdom). The study was conducted in accordance with the prin- ciples of Good Clinical Practice and the World Medical Association Declaration of Helsinki (1964) and its amendments and subsequent clarifications. The relevant local ethics committees approved the study and written informed consent was obtained from patients and caregivers before enrolment. Due to ethical considerations (availability of efficient symptomatic drugs for $\mathrm{AD}$ in the majority of countries), a majority of investigators required to minimise patient exposure to placebo. Therefore, eligible patients were randomised in a 2:1 ratio to 3 weeks of up-titration and 21 weeks of treatment with memantine $(20 \mathrm{mg} /$ day $)$ or placebo, respectively. Dosing of memantine was initiated with $5 \mathrm{mg} /$ day and titrated up with weekly steps to $20 \mathrm{mg} /$ day (10 mg twice daily) according to the current Summary of Product Characteristics. The first patient first visit was in May 2002 and the last patient last visit was in September 2003. H. Lundbeck A/S funded the study and was involved in planning the design, the protocol and data analysis along with the author(s). The data are stored at $\mathrm{H}$. Lundbeck A/S.

\section{Patients}

Patients who fulfilled the diagnosis of probable AD according to National Institute of Neurological Disorders and Stroke-Alzheimer Disease and Related Disorders Association criteria [15] and with the Diagnostic and Statistical Manual of Mental Disorders, Fourth Edition criteria for dementia of the Alzheimer's type, including a computer tomography or magnetic resonance imaging of the brain within the previous 12 months with results consistent with such diagnosis were eligible for inclusion if they were an outpatient of either sex, more than 50 years old and had a baseline MMSE total score between 11 and 23 (extremes included) [6]. Furthermore, the patients should have had a reliable and knowledgeable caregiver who could accompany the patient to all clinic visits during the study. Patients with vascular dementia, dementia or clinically significant neurological disease other than Alzheimer's disease, major depressive disorder or a score greater than 4 on the modified Haskinski Ischemic Rating Scale [30] were excluded. Patients with clinically significant coexisting medical conditions or laboratory abnormalities were also excluded, as were patients receiving specific concomitant medications (anticonvulsants, antiparkinson agents, classical and depot antipsychotics, anxiolytics, hypnotics, non-SSRI antidepressants, cholinesterase inhibitors or any other investigational product). When used previously to in- 
clusion, cholinesterase inhibitors were discontinued at least 30 days prior to the screening visit. SSRIs, estrogens, antiinflammatory drugs, $\beta$-blockers, insulin and $\mathrm{H}_{2}$ blockers were allowed if dose and medication had been stable for at least 3 months and were kept stable during the study. Vitamin E, Coenzyme Q and atypical antipsychotics (risperidone $\leqslant 2 \mathrm{mg} /$ day, olanzapine $\leqslant 10 \mathrm{mg} /$ day or quetiapine $\leqslant 100 \mathrm{mg} /$ day) were allowed if dose and medication had been stable for at least 30 days and kept stable during the study. Atypical antipsychotics were not to be taken 3 days before a visit. Only non-opioid analgesics could be administered chronically.

A patient was withdrawn from the study if the randomisation code for that patient was broken, or if consent to participate was withdrawn for the patient or caregiver. A patient could also be withdrawn from the study if: they had a serious adverse event (SAE: death, life-threatening condition, hospitalisation), the caregiver became unavailable, the patient was lost to follow-up, or the patient was placed in a nursing home.

\section{Primary efficacy variables}

Patients were assessed at screening, at baseline and 4, 12, 18 and 24 weeks after the start of double-blind treatment. The pre-specified primary efficacy variables were change from baseline on Alzheimer's Disease Assessment Scale - Cognitive Subscale (ADAS-cog) total score [29] at week 24 and Clinician's Interview-Based Impression of Change Plus Caregiver Input (CIBICplus) total score [24] at week 24. The ADAS-cog is an 11-item scale assessing cognitive domains of memory, language, orientation, reasoning, and praxis. Scores range from 0 to 70 with lower scores indicating less impairment. Its use in measuring changes in cognition in mild to moderate AD has been extensively validated $[16,19,34]$. The CIBIC-Plus is used to assess treatment effects on overall clinical status in $\mathrm{AD}$, based on an independent comprehensive interview with the participant and caregiver by an experienced rater/clinician. Scores on the CIBIC-Plus range from 1 (marked improvement) to 7 (marked worsening). A score of 4 represents no change. The CIBIC-Plus score is assigned at all post-baseline visits relative to a global assessment of disease severity made at baseline. Given the multilingual nature of this study, translated versions of this scale were used to match the native language at the location of each centre.

\section{Secondary efficacy variables}

Secondary efficacy measures included Neuropsychiatric Inventory (NPI) [4] and the 23-item Alzheimer's Disease Cooperative Study Activities of Daily Living Inventory (ADCS-ADL 23 ) scale [9]. Patients were assessed on the NPI and the ADCS-ADL 23 at baseline and weeks 12 and 24. The NPI is a 12-item, caregiver interview-based instrument designed to assess frequency and severity of behavioural symptoms in dementia patients. The NPI provides both a total score (ranging from 0 to 144) as well as scores for each of its 12 items; higher scores reflect greater symptom severity. For each item, the frequency and severity of each behaviour is measured. The ADCS-ADL 23 for mild to moderate $\mathrm{AD}$ patients consists of 23 items used to assess basic and instrumental activities of daily living (ADLs). Most ADL items are comprised of a series of hierarchical sub-questions, with possible scores ranging from 0 to 78 reflecting the complete loss of ability to perform the ADL to the highest level of independent performance. Higher scores indicate better performance. The ADCS-ADL 23 was administered as an interview with the caregiver and assessed ADL over the previous four weeks.

\section{Power and sample size calculations}

The sample size calculation was based on the success criterion of the medication having an effect on the primary efficacy variables applying a 5\% significance level for each analysis. Assuming an effect size of 0.43 for ADAS-cog total scores, 0.49 for CIBIC-plus rating and a sample size of 400 patients (133 in the placebo group and 267 in the memantine group), the study had a power of $88 \%$ to detect a significant difference between the treatments.

Rater training was undertaken to increase inter-rater reliability. Only personnel that had actively participated in rater training sessions prior to enrolment of patients were allowed to rate patients. The rater who scored the CIBIC-plus was blinded to the results of the other efficacy assessments. The same rater was to score the patient throughout the study if possible. The training materials for efficacy assessment were translated for the raters and back-translated and medically verified. 


\section{Allocation to treatment}

The study products were tablets of identical appearance, taste and smell. Patients were assigned to 24weeks of double-blind treatment with memantine or placebo in a 2:1 ratio. The details of the randomisation series were unknown to any of the investigators and were contained in a set of sealed opaque envelopes. At each study centre, sequentially enrolled patients were assigned the lowest randomisation number available. All study personnel and participants were blinded to treatment assignment for the duration of the study.

\section{Primary efficacy assessments}

The following populations were considered in the statistical analyses: the all patients treated set (APTS), which consisted of all randomised patients who took at least one dose of study product and was identical with the safety population (a total of 470 patients; 318 treated with memantine and 152 treated with placebo). The full analysis set (FAS) consisted of all patients in the APTS who had at least one valid post-baseline assessment of both primary efficacy parameters (a total of 461 patients; 310 treated with memantine and 151 treated with placebo) and the 24 week completers set (CS24) consisted of all patients in the FAS who took randomised study product up to week 24 and were assessed on both primary efficacy parameters at the 24 week visit (a total of 403 patients; 268 treated with memantine and 135 treated with placebo). The primary efficacy variables (ADAS-cog and CIBIC-plus change from baseline in the CS24) were analysed using analysis of covariance (ANCOVA) with treatment and centre as factors and the relevant baseline value as a covariate. Two-sided hypothesis testing was performed at the 5\% significance level. Results were summarised using the least squares mean (LS mean) for each treatment group with corresponding standard errors.

\section{Secondary efficacy assessments}

The secondary efficacy variables (change from baseline in NPI and ADCS-ADL 23 in the CS24) were analysed using ANCOVA with treatment and centre as factors and the relevant baseline value as a covariate. They were analysed per visit for both observed cases (OC) and last observation carried forward (LOCF). Twosided hypothesis testing was performed at the $5 \%$ significance level.
As a sensitivity analysis, the CIBIC-plus was analysed at the last visit using the Cochran-MantelHaenszel $(\mathrm{CMH})$ test stratifying by centre.

The proportions of responders based on the CS24 were analysed per visit using Fisher's Exact test. Responders were defined as patients showing marked improvement in cognitive function (change in ADAS-cog $\leqslant-4)$ and global improvement or stabilisation (CIBICplus $\leqslant 4)$. No interim analyses were performed. SAS version 6.12 (SAS institute, Cary, NC) was used for all analyses.

\section{Safety and Tolerability Assessments}

The safety population was identical to the APTS. Safety and tolerability were evaluated on the basis of adverse events (AEs), clinical laboratory safety data, vital signs, ECGs and weight. AE data and vital signs were collected at every visit; clinical laboratory safety data and ECGs were assessed at screening and week 24. A final safety assessment was made 30 days after the last assessment of double-blind treatment. For safety analyses, an AE occurring during the double-blind treatment period was considered a treatment-emergent AE (TEAE) if it was not present prior to the start of the double-blind medication or if it was present at baseline but increased in severity during the double-blind treatment period. The incidence of individual TEAEs was compared between treatment groups using Fisher's Exact test.

\section{RESULTS}

Patient disposition, demographics and baseline characteristics; Study 99679

A total of 470 patients were randomised and treated (memantine: 318, placebo: 152; Table 1). Of these patients, $268(84 \%)$ in the memantine group and 135 $(89 \%)$ in the placebo group were included in the CS24. The two groups were comparable with respect to proportion of women/men and age. Women constituted $65 \%$ of the patients and the mean age was 74 years (ranging from 54 to 89 years). The two treatment groups were also comparable with respect to the severity of AD at baseline. The mean MMSE total scores were $18.6 \pm 3.3$ for memantine and $18.9 \pm 3.2$ for placebo treated patients. The mean ADAS-cog total scores at baseline were $25.9 \pm 10.4$ for the memantine and $24.9 \pm 9.7$ for placebo treated patients. This is in- 
Table 1

Disposition, demographics and mean baseline scores

\begin{tabular}{lcc}
\hline & Memantine & Placebo \\
\hline Disposition & & \\
Patients randomised: $\mathrm{n}$ & 318 & 152 \\
Patients treated (APTS): $\mathrm{n}$ & 318 & 152 \\
Patients withdrawn: n (\%) & $47(15)$ & $14(9)$ \\
Full analysis set (FAS): n (\%) & $310(97)$ & $151(99)$ \\
Patients completed: n (\%) & $271(85)$ & $138(91)$ \\
Completers set (CS24): n (\%) & $268(84)$ & $135(89)$ \\
Demographics & & \\
Female: n (\%) & $206(65)$ & $91(60)$ \\
Mean age (years) \pm SD & $74.0 \pm 7.4$ & $73.3 \pm 6.9$ \\
Mean weight (kg) \pm SD & $65.8 \pm 12.2$ & $66.2 \pm 11.5$ \\
Caucasian: $\mathrm{n}(\%)$ & $318(100)$ & $152(100)$ \\
Baseline scores & & \\
Mean MMSE score \pm SD & $18.6 \pm 3.3$ & $18.9 \pm 3.2$ \\
Mean ADAS-cog score \pm SD & $25.9 \pm 10.4$ & $24.9 \pm 9.7$ \\
Previous AChEI usage: $\mathrm{n}(\%)$ & $121(38)$ & $54(36)$ \\
\hline
\end{tabular}

dicative of a population with mild to moderate AD. The proportions of patients previously treated with AChEIs were $38 \%$ in the memantine group and $36 \%$ in the placebo group.

The proportion of patients who withdrew from the study was low: $15 \%$ of the patients in the memantine group versus $9 \%$ in the placebo group (Fig. 1). The most common reasons for withdrawal were AEs (9\% in the memantine group versus $4 \%$ in the placebo group) and withdrawal of consent (2.5\% in the memantine group versus $2 \%$ in the placebo group). No trends were seen in the AEs that resulted in withdrawal. In the memantine group, 4 patients withdrew due to dizziness and 3 patients withdrew due to depression. In the placebo group, none of the AEs leading to withdrawal were reported by more than one patient.

\section{Efficacy of memantine; Primary endpoints}

The effect of memantine compared to placebo in the CS24 on the ADAS-cog and CIBIC-plus scales is shown in Fig. 2 and Table 2. The scores for memantinetreated patients on both the ADAS-cog and CIBIC-plus scales were numerically better than those for placebo treated patients at all post-baseline assessments. Statistically significant separation in favour of memantine was seen at weeks 12 and 18 on both scales. However, on the predefined primary efficacy endpoint (i.e., at week 24), the difference in favour of memantine was not statistically significant. Nevertheless, memantinetreated patients showed a mean improvement of 1.93 points at week 24 on the ADAS-cog scale as compared to a 1.08 points improvement in placebo-treated patients.
The proportion of patients who responded to treatment was numerically greater in the memantine group compared to the placebo group at all assessments (Fig. 3, Table 3). Statistically significant separation in favour of memantine was seen at weeks 12 and 18 .

\section{Efficacy of memantine; Secondary endpoints}

The effect of memantine compared to placebo in the CS24 on the NPI and ADCS-ADL 23 scale is shown in Table 2. The NPI total score improved in both treatment groups over time but there was no statistically significant difference between the memantine and placebo group. Likewise, no significant difference between the memantine and placebo group was detected in the ADCS-ADL 23 total score.

\section{Safety and tolerability}

Approximately half of all patients experienced TEAEs. The incidence of TEAEs was similar in both treatment groups and was very low given the patient population and the duration of study. The adverse events with an incidence of $4 \%$ in either group are summarised in Table 4. In both treatment groups, the majority of the TEAEs were considered by the investigator to be mild to moderate.

A total of 8 patients died during the study or within 30 days after the last dose of study drug (one during screening, 5 during double-blind memantine treatment, one during double-blind placebo treatment and one placebo-treated patient after withdrawal). None of the events leading to death were considered related to treatment. A total of 42 patients had SAEs after the 
Table 2

Results of the efficacy parameters by study week on the CS24 (OC)

\begin{tabular}{|c|c|c|c|c|c|c|c|}
\hline \multirow[t]{2}{*}{ Outcome measure } & \multirow[t]{2}{*}{ Week } & \multicolumn{2}{|c|}{ Memantine } & \multicolumn{2}{|r|}{ Placebo } & \multicolumn{2}{|c|}{ Difference from placebo } \\
\hline & & $\mathrm{N}$ & $\begin{array}{c}\text { Change from } \\
\text { baseline }\end{array}$ & $\mathrm{N}$ & $\begin{array}{c}\text { Change from } \\
\text { baseline }\end{array}$ & Mean $[95 \% \mathrm{CI}]$ & $\begin{array}{c}\text { ANCOVA } \\
\text { p-value }\end{array}$ \\
\hline \multirow{4}{*}{ ADAS-cog } & 4 & 267 & -1.71 & 135 & -1.61 & $-0.10[-0.98,0.78]$ & 0.827 \\
\hline & 12 & 268 & -2.46 & 135 & -0.70 & $-1.76[-2.69,-0.83]$ & 0.000 \\
\hline & 18 & 266 & -2.26 & 135 & -0.98 & $-1.29[-2.33,-0.25]$ & 0.016 \\
\hline & $24^{\mathrm{a}}$ & 268 & -1.93 & 135 & -1.08 & $-0.85[-2.02,0.32]$ & 0.156 \\
\hline \multirow[t]{4}{*}{ CIBIC-plus } & 4 & 266 & 3.94 & 134 & 3.95 & $-0.01[-0.16,0.14]$ & 0.896 \\
\hline & 12 & 267 & 3.90 & 134 & 4.11 & $-0.21[-0.40,-0.02]$ & 0.033 \\
\hline & 18 & 268 & 3.99 & 135 & 4.27 & $-0.28[-0.49,-0.06]$ & 0.012 \\
\hline & $24^{\mathrm{a}}$ & 268 & 4.12 & 135 & 4.19 & $-0.07[-0.30,0.15]$ & 0.523 \\
\hline \multirow[t]{2}{*}{$\mathrm{ADCS}_{-\mathrm{ADL}_{23}}$} & 12 & 268 & -0.67 & 135 & -0.19 & $-0.48[-1.80,0.85]$ & 0.480 \\
\hline & 24 & 267 & -1.99 & 134 & -2.08 & $0.09[-1.52,1.70]$ & 0.912 \\
\hline \multirow[t]{2}{*}{ NPI } & 12 & 268 & -1.37 & 135 & -1.02 & $-0.35[-1.96,1.26]$ & 0.671 \\
\hline & 24 & 267 & -1.45 & 134 & -2.73 & $1.28[-0.50,3.05]$ & 0.159 \\
\hline
\end{tabular}

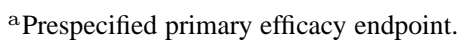

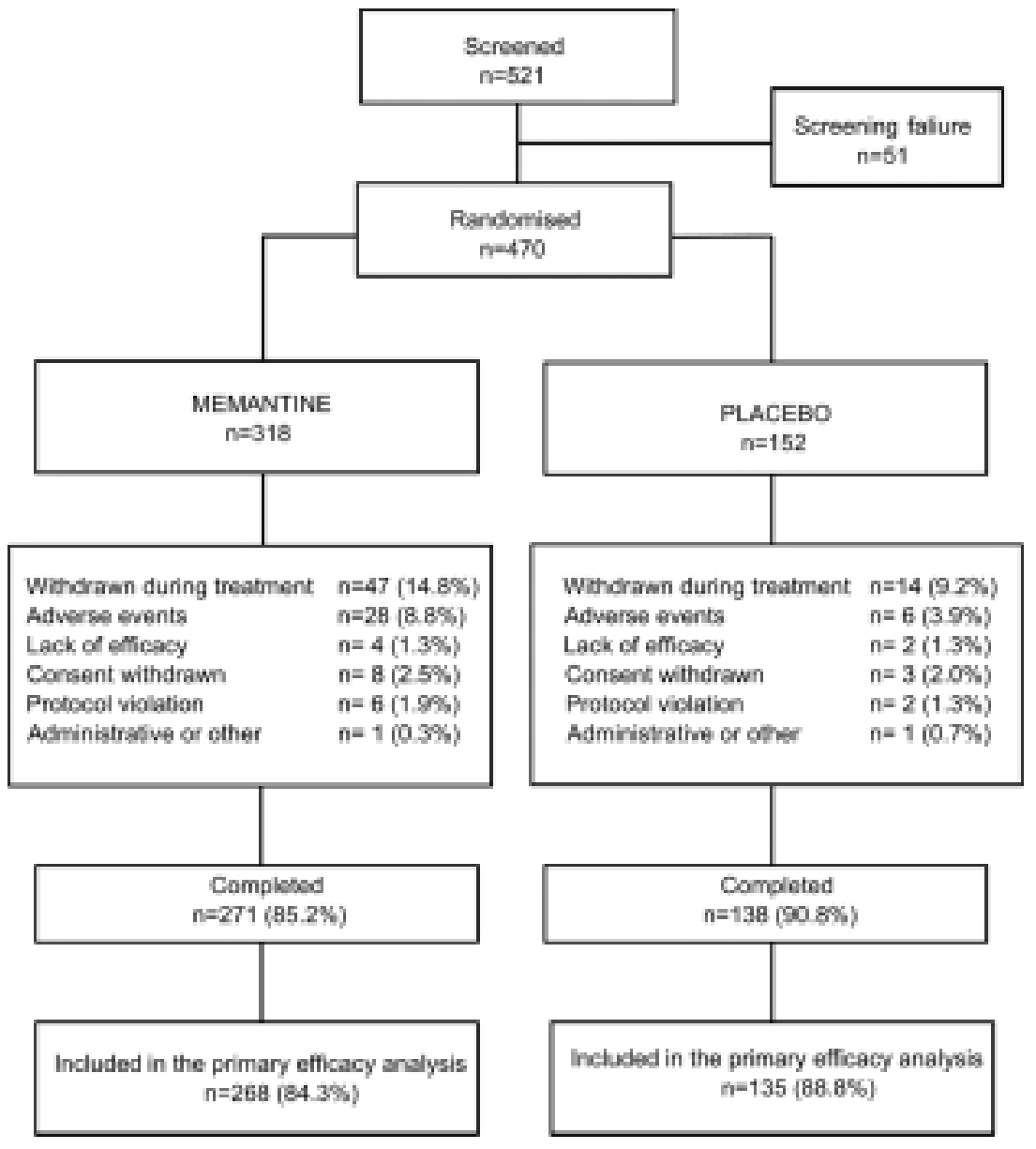

Fig. 1. Patient disposition

start of the treatment (10\% in the memantine group and $6 \%$ in the placebo group). SAEs reported in 2 or more participants in either memantine or placebo group were accidental injury (memantine: 5, placebo: 0), depression (memantine: 3 , placebo: 0 ), pneumonia (memantine: 2, placebo: 1), cerebral haemorrhage (meman- 

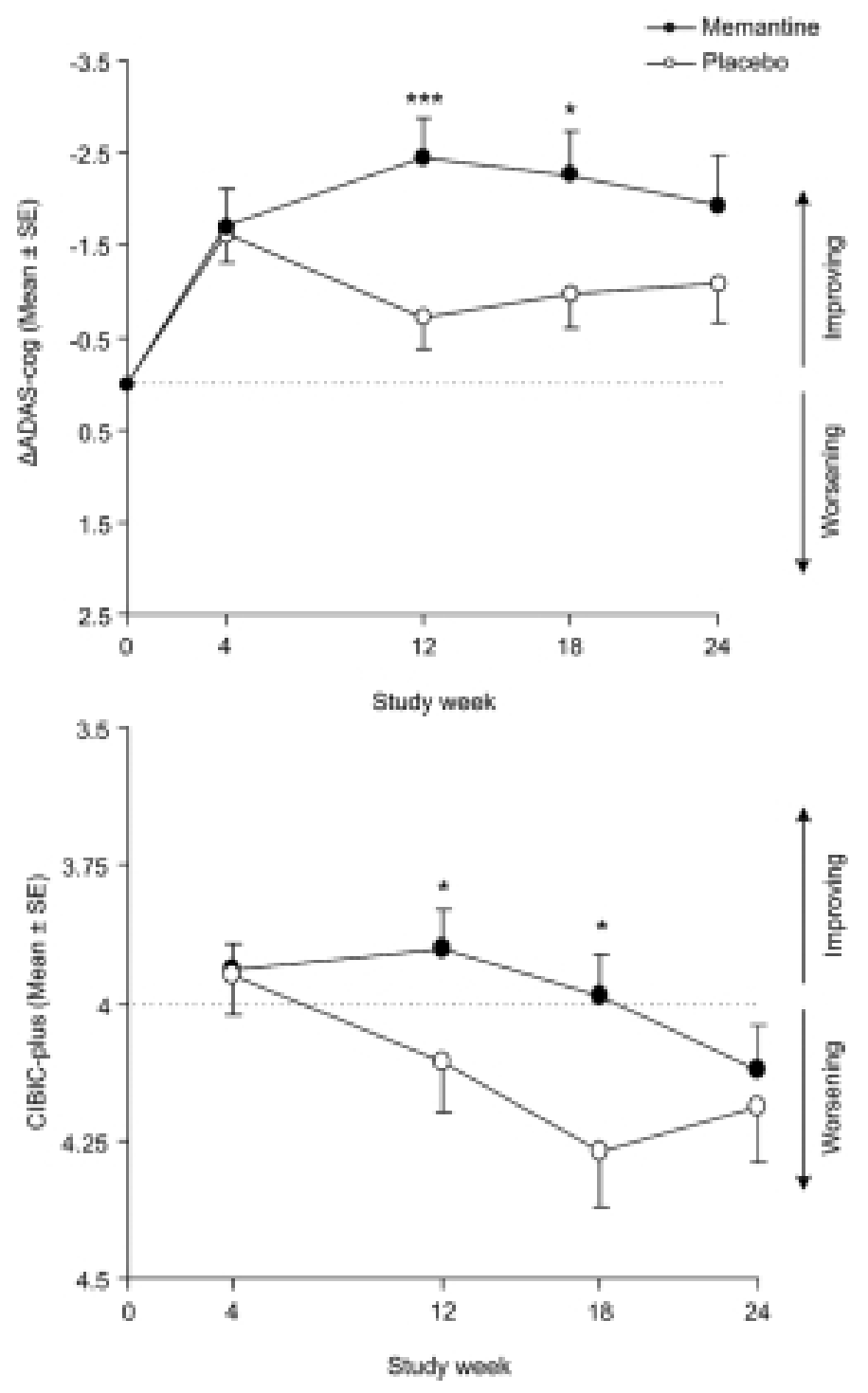

Fig. 2. Results of the primary efficacy parameters (ADAS-cog change from baseline score and CIBIC-plus total score) for memantine and placebo based on the CS24. Error bars indicate standard error (SE). ${ }^{*} p<0.05,{ }^{* * *} p<0.001$ compared to placebo (ANCOVA).

tine: 2, placebo: 0), abdominal pain (memantine: 2, placebo: 0) and myocardial infarction (memantine: 2 , placebo: 1). Three patients had an SAE that was considered by the investigator to be possibly or probably related to treatment. These events were fall, accidental injury and agitation (all belonging to the memantine group). The remaining SAEs were all considered to be unrelated to treatment.

The incidence of potentially clinically significant laboratory values, vital signs and ECG values was low. There were no clinically meaningful differences between treatment groups in the mean change from base- line to endpoint for laboratory tests, vital signs, weight or ECG parameters.

\section{DISCUSSION}

The present placebo-controlled, 6-month study (99679) in outpatients with mild to moderate AD indicates that memantine is safe and well tolerated. The pattern of adverse events in the memantine group was similar to that experienced by the patients in the placebo group. The high study completion rate in the memantine group (around 85\%) and the low level of ad- 
Table 3

Proportion of responders by study week based on the CS24 (OC)

\begin{tabular}{|c|c|c|c|c|c|}
\hline \multirow[t]{2}{*}{ Week } & \multicolumn{2}{|r|}{ Memantine } & \multicolumn{2}{|r|}{ Placebo } & \multirow{2}{*}{$\begin{array}{c}\text { Difference from placebo } \\
\text { Fisher's Exact test } \\
\text { p-value }\end{array}$} \\
\hline & $\mathrm{n}$ & Responders $^{\mathrm{a}}(\%)$ & $\mathrm{n}$ & Responders $^{\mathrm{a}}(\%)$ & \\
\hline 4 & 265 & 22.3 & 134 & 20.1 & 0.700 \\
\hline 12 & 267 & 30.0 & 134 & 15.7 & 0.002 \\
\hline 18 & 266 & 30.1 & 135 & 20.0 & 0.032 \\
\hline 24 & 268 & 31.3 & 135 & 25.2 & 0.246 \\
\hline
\end{tabular}

${ }^{\text {a }}$ Responders were defined as patients showing marked improvement in cognitive function (change in ADAS-cog $\leqslant-4$ ) and global stabilisation or improvement (CIBIC-plus $\leqslant 4$ ).

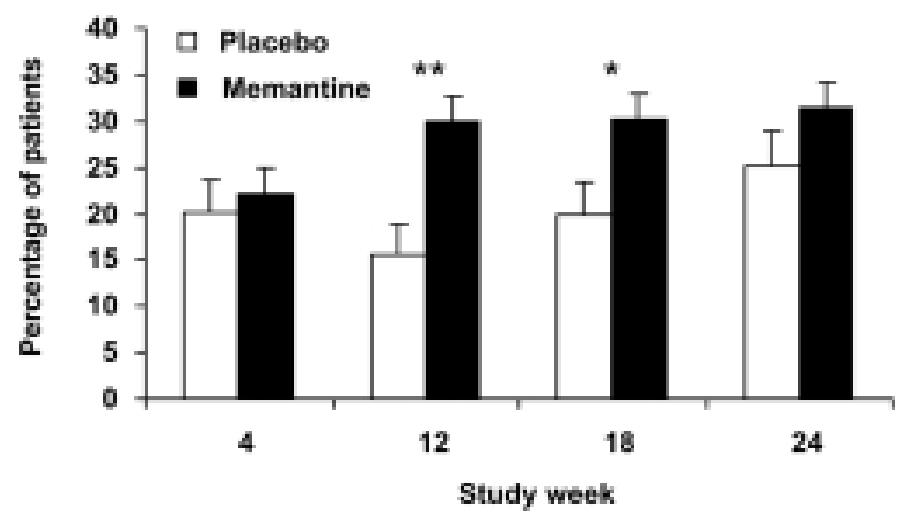

Fig. 3. Proportion of responders in patients treated with memantine or placebo based on the CS24. Responders were defined as patients showing marked improvement in cognitive function (change in ADAS-cog $\leqslant-4$ ) and global stabilisation or improvement (CIBIC-plus $\leqslant 4) .{ }^{*} p<0.05$, ${ }^{* *} p<0.01$ compared to placebo (Fisher's Exact test).

verse events observed in this study are consistent with previous studies demonstrating an excellent safety and tolerability profile of memantine in $\mathrm{AD}[25,26,20,33]$.

On the primary efficacy variables (ADAS-cog and CIBIC-plus) memantine separated from placebo early on and was statistically superior to placebo at study weeks 12 and 18 on a $95 \%$ significance level. Although numerically superior, the benefit of memantine was not statistically significant at the predefined primary efficacy endpoints, i.e., change from baseline on ADAS-cog total score and CIBIC-plus total score at study week 24. Memantine treatment was associated with significant and clinically relevant cognitive improvements above baseline throughout the entire study indicating an overall maintenance of cognitive function from study start to end point. This conclusion is supported by the fact that the analysis was made on the CS24 and not based on a LOCF approach where early drop-outs may falsely indicate a maintenance of cognitive function given the degenerative nature of this disease. Other studies in mild to moderate AD or vascular dementia have shown that memantine is efficaceous in reducing the deterioration assess by the ADAS-cog $[17,20,35]$.
Table 4

Treatment-emergent adverse events (TEAEs) with an incidence of $\geqslant 4 \%$ in one of the treatment groups in the APTS

\begin{tabular}{lcc}
\hline Preferred term & $\begin{array}{c}\text { Memantine } \\
(n=318)\end{array}$ & $\begin{array}{c}\text { Placebo } \\
(n=152)\end{array}$ \\
& 56.0 & 52.6 \\
\hline Patients with TEAEs & 5.7 & 2.0 \\
Headache & 5.3 & 3.9 \\
Dizziness & 5.0 & 5.3 \\
Accidental/inflicted injury & 4.4 & 4.6 \\
Rhinitis & 1.6 & 4.6 \\
Agitation & & \\
\hline
\end{tabular}

The lack of statistically significant results for the primary efficacy analyses at study week 24 , after being statistically significantly superior to placebo at study week 12 and 18, was unexpected. This lack of a statistically significant difference may be attributed to an atypical placebo response, that is, the placebo group did not deteriorate over the 24 weeks study period as expected for this patient population. The patients receiving placebo showed an unexpected improvement on both primary efficacy scales late in the study.

There are several published placebo-controlled clinical studies in dementia using a similar patient population that support the notion that the observed placebo 
response of the present study was indeed unusual [20, 23]. In a recent published study, the effect of 1-year treatment with the anti-inflammatory agent rofecoxib was tested in 692 patients with mild to moderate AD and a gradual decline relative to baseline was observed during the study [23]. After 24 weeks of treatment the mean deterioration in the placebo group on the ADAScog scale was around 2.2 points which is similar to the degree of deterioration observed in the earlier placebocontrolled studies with AChEIs [5,28,29,31]. This is in clear contrast to the 1.08 points improvement seen in this study.

The reason for this unusual placebo response was investigated but neither compromised blinding, vascular comorbidity, previous AChEI treatment nor concomitant medication could account for the observed effect. An analysis to investigate the treatment-by-centre interaction identified a heterogeneous treatment effect over centres $(p=0.056)$ for the CIBIC-plus scores.

A measured point difference between placebo- and drug-treated patients on a quantitative scale may not necessarily represent a clinically meaningful improvement. Responder analyses (rates of individual response) are often performed as an indication of the clinical relevance of a treatment.

Responder rates were significantly better in the memantine group compared to the placebo group at study weeks 12 and 18, but not at week 24. This finding is noteworthy for two reasons. First, the current study was not powered to show a statistically significant separation on categorical data. Second, it is unusual for a single study in mild to moderate AD to show a statistically significant difference from placebo using a conservative dual-responder criterion. The lack of statistical significance at week 24 can be attributed to an increase in the number of placebo responders. The number of memantine responders remained generally stable.

Previous studies have provided evidence that memantine is efficacious in moderate to severe $\operatorname{AD}[25,26$, $33,37]$. The results presented and discussed in this paper extend these findings by indicating that modulation of NMDA receptor-mediated neurotransmission is also an attractive approach in the treatment of mild to moderate AD especially when taken together with recently published results of another mild to moderate AD study [20]. The efficacy of memantine across all severity stages of $\mathrm{AD}$ is consistent with previous findings indicating the presence of glutamatergic abnormalities at all stages of this disease [7,10,21].

\section{ACKNOWLEDGEMENTS}

We gratefully acknowledge the participating patients and their caregivers. We also thank the following investigators in each of the 65 primary care centres for their contribution to this study: Austria: Manfred Haushofer, Josef Marksteiner, Peter Dal-Bianco, Reinhold Schmidt, Friedrich Leblhuber, Michael Rainer; Belgium: Maurits Vandewoude, Patrick Cras, Rik Vandenberghe, Eric Salmon, Peter Paul De Deyn; Denmark: Gunhild Waldemar, Jørgen Jensen, Hans Brændgaard, Per Kragh-Sørensen; Finland: Tuula Pirttilä, Kari Alhainen, Keijo Koivisto, Riitta Niskanen; France: Jacques Boulliat, René Decombe, Olivier Moreaud, Michel Poncet, Jacques Louvrier, Michèle Kiesmann, Florence Mahieux-Laurent, Jean-Philippe Chartier, Claude-Francois Degos, Philippe LebrunGrandié, Nicolas Schmidt, Josette Leche, Antoine Engles, Pierre De Facq, Jacques Touchon, François Blanchard, Jean-Louis Albarède, Pierre Parry, Serge Belliard, Pierre Haond; Greece: Klimentini Karageorgiou, Paraskevi Sakka, Athanase Mouyas/Irena Kyriakopoulou; Lithuania: Valmantas Budrys, Vanda Liesene; The Netherlands: Philip Scheltens, Frans Verhey; Poland: Iwona Kloszewska, Wojciech Kozubski, Andrzej Szcsudlik, Maria-Vanda Barcikowska, Grzegorz Opala; Spain: Miguel Aguilar, Josep M. Manubens, Secundino López-Pousa, Jaime Kulisevsky, Mercè Boada, Rafael Blesa, Félix Bermejo, Maria-Camino Sevilla, Román Alberca, Ramon Reñé, Ana Frank, Jordi Peña-Casanova, Oscar Fernández; Sweden: Anders Wallin, Niels Andreasen, Lennart Minthon, Sture Eriksson; The United Kingdom: Roger Bullock, Roy Jones, David Wilkinson, Anthony Bayer. This study was sponsored by H. Lundbeck A/S, who also provided an honoraria to Prof. Bakchine, as main investigator.

\section{References}

[1] A. Burns, M. Rossor, J. Hecker, S. Gauthier, H. Petit, H.J. Möller and the International Donepezil Study Group, The effects of donepezil in Alzheimer's disease - Results from a Multinational Trial, Dement Geriatr Cogn 10 (1999), 237244.

[2] J. Corey-Bloom, R. Anand and J. Veach, for the ENA 713 B352 study group, A randomized trial evaluating the efficacy and safety of ENA 713 (rivastigmine tartrate), a new acetylcholinesterase inhibitor, in patients with mild to moderately severe Alzheimer's disease, Int J Geriatr Psychopharmacol 1 (1998), 55-65.

[3] J.L. Cummings and G. Cole, Alzheimer disease, JAMA 287 (2002), 2335-2338. 
[4] J.L. Cummings, M. Mega, K. Gray, S. Rosenberg-Thompson, D.A. Carusi and J. Gornbein, The Neuropsychiatric Inventory: comprehensive assessment of psychopathology in dementia, Neurology 44 (1994), 2308-2314.

[5] M. Farlow, R. Anand, J. Messina, Jr., R. Hartman and J. Veach, A 52-week study of the efficacy of rivastigmine in patients with mild to moderately severe Alzheimer's disease, Eur Neurol 44 (2000), 236-241

[6] M.F. Folstein, S.E. Folstein and P.R. McHugh, Mini-Mental state. A practical method for grading the cognitive state of patients for the clinician, J Psychiat Res 12 (1975), 189-198.

[7] P.T. Francis, N.R. Sims, A.W. Procter and D.M. Bowen, Cortical pyramidal neurone loss may cause glutamatergic hypoactivity and cognitive impairment in Alzheimer's disease: investigative and therapeutic perspectives, J Neurochem 60 (1993), 1589-1604.

[8] L. Fratiglioni, D. De Ronchi and H. Aguero-Torres, Worldwide prevalence and incidence of dementia, Drug Aging $\mathbf{1 5}$ (1999), 365-375.

[9] D. Galasko, D. Bennett, M. Sano, C. Ernesto, R. Thomas, M. Grundman and the Alzheimer's Disease Cooperative Study, An inventory to assess activities of daily living for clinical trials in Alzheimer's disease. The Alzheimer's Disease Cooperative Study, Alz Dis Assoc Dis 11 (1997), S33-S39.

[10] N. Hattori, K. Abe, S. Sakoda and T. Sawada, Proton MR spectroscopic study at 3 Tesla on glutamate/glutamine in Alzheimer's disease, Neurorepor 13 (2002), 183-186.

[11] A.F. Jorm and D. Jolley, The incidence of dementia: a metaanalysis, Neurology 51 (1998), 728-733.

[12] J. Kornhuber and M. Weller, Psychotogenicity and N-methylD-aspartate receptor antagonism: implications for neuroprotective pharmacotherapy, Biol Psychiatry 41 (1997), 135-144.

[13] G. Livingston and C. Katona, The place of memantine in the treatment of Alzheimer's disease: a number needed to treat analysis, Int J Geriatr Psychiatry 19 (2004), 919-925.

[14] A. Lobo, L.J. Launer, L. Fratiglioni, K. Andersen, A. Di Carlo, M.M. Breteler, J.R. Copeland, J.F. Dartigues, C. Jagger, J Martinez-Lage, H. Soininen, A. Hofman and the Neurologic Diseases in the Elderly Research Group, Prevalence of dementia and major subtypes in Europe: A collaborative study of population-based cohorts, Neurology 54 (2000), S4-S9.

[15] G. McKhann, D. Drachman, M. Folstein, R. Katzman, D. Price and E.M. Stadlan, Clinical diagnosis of Alzheimer's disease: report of the NINCDS-ADRDA Work Group under the auspices of Department of Health and Human Services Task Force on Alzheimer's Disease, Neurology 34 (1984), 939-944.

[16] B.M. McLendon and P.M. Doraiswamy, Defining meaningful change in Alzheimer's disease trials: the donepezil experience, J Geriat Psychiatry Neurol 12 (1999), 39-48.

[17] J.M. Orgogozo, A.S. Rigaud, A. Stöffler, H.J. Möbius and F. Forette, Efficacy and safety of memantine in patients with mild to moderate vascular dementia: a randomized, placebocontrolled trial (MMM300), Stroke 33 (2002), 1834-1939.

[18] C.G. Parsons, W. Danysz and G. Quack, Memantine is a clinically well tolerated N-methyl-D-aspartate (NMDA) receptor antagonist: A review of preclinical data, Neuropharmacolog 38 (1999), 735-767.

[19] J. Pena-Casanova, Alzheimer's Disease Assessment Scalecognitive in clinical practice, Inl Psychogeriat 9 (1997), S105S114.

[20] E.R. Peskind, S.G. Potkin, N. Pomara et al., Memantine treatment in mild to moderate Alzheimer disease: a 24-week randomized controlled trial, Am J Geriatr Psychiatry 14 (8) (2006), 704-715

[21] N. Pomara, R. Singh, D. Deptula, J.C. Chou, M.B. Schwartz and P.A. LeWitt, Glutamate and other CSF amino acids in Alzheimer's disease, Am J Psychiatry 49 (1992), 251-254.

[22] M.A. Raskind, E.R. Peskind, T. Wessel, W. Yuan and The Galantamine USA-1 Study Group, Galantamine in AD: A 6month randomized, placebo-controlled trial with a 6-month extension, Neurology 54 (2000), 2261-2268.

[23] S.A. Reines, G.A. Block, J.C. Morris, G. Liu, M.L. Nessly, C.R. Lines, B.A. Norman, C.C. Baranak and the Rofecoxib Protocol 091 Study Group, Rofecoxib: no effect on Alzheimer's disease in a 1-year, randomized, blinded, controlled study, Neurology 62 (2004), 66-71.

[24] B. Reisberg, L. Schneider, R. Doody, R. Anand, H. Feldman and H. Haraguchi, Clinical global measures of dementia: Position paper from the International Working Group on Harmonization of Dementia Drug Guidelines, Alz Dis Assoc Dis 11 (1997), S8-S18.

[25] B. Reisberg, R. Doody, A. Stoffler, F. Schmitt, S. Ferris and H.J. Mobius, A 24-week open-label extension study of memantine in moderate to severe Alzheimer disease, Arch Neur 63 (2006), 49-54.

[26] B. Reisberg, R. Doody, A. Stöffler, F. Schmitt, S. Ferris, H.J. Möbius, Memantine in moderate-to-severe Alzheimer's disease, $N$ Engl J Med 348 (2003), 1333-1341.

[27] M.A. Rogawski and G.L. Wenk, The neuropharmacological basis for the use of memantine in the treatment of Alzheimer's disease, CNS Drug Rev 9 (2003), 275-308.

[28] S.L. Rogers, M.R. Farlow, R.S. Doody, R. Mohs, L.T. Friedhoff and the Donepezil Study Group, A 24-week, doubleblind, placebo-controlled trial of donepezil in patients with Alzheimer's disease, Neurology 50 (1998), 136-145.

[29] W.G. Rosen, R.C. Mohs and K.L. Davis, A new rating scale for Alzheimer's Disease, Am J Psychiatry 141 (1984), 13561364.

[30] W.G. Rosen, R.D. Terry, P.A. Fuld, R. Karzman and A. Peck, Pathologic verification of ischemic score in differentiation of dementias, Ann Neurol 5 (1980), 486-488.

[31] M. Rösler, R. Anand, A. Cicin-Sain, S. Gauthier, Y. Agid, P. Dal-Bianco, H.B. Stahelin, R. Hartman and M. Gharabawi on behalf of the B303 Exelon Study Group. Efficacy and safety of rivastigmine in patients with Alzheimer's disease: international randomized controlled trial, BMJ 318 (1999), 633-638.

[32] D.J. Selkoe, The molecular pathology of Alzheimer's disease, Neuron 6 (1991), 487-498.

[33] P.N. Tariot, M.R. Farlow, G.T. Grossberg, S.M. Graham, S. McDonald and I. Gergel, Memantine treatment in patients with moderate to severe Alzheimer disease already receiving donepezil: a randomized controlled trial, JAMA 291 (2004), 317-324.

[34] G. Weyer, H. Erzigkeit, S. Kanowski, R. Ihl and D. Hadler. Alzheimer's Disease Assessment Scale: reliability and validity in a multicenter clinical trial, Int Psychogeriatr 9 (1997), 123-138.

[35] G. Wilcock, H.J. Möbius and A. Stöffler, mmm 500group, A double-blind, placebo-controlled multicentre study of memantine in mild to moderate vascular dementia (MMM500), Int Clin Psychopharmacol 17 (2002), 297-305.

[36] G.K Wilcock, S. Lilienfeld and E. Gaens on behalf of the Galantamine International-1 Study Group, Efficacy and safety of galantamine in patients with mild to moderate Alzheimer's disease: multicentre randomised controlled trial, BMJ $\mathbf{3 2 1}$ (2000), 1445-1449. 
[37] B. Winblad and N. Poritis, Memantine in severe dementia: results of the M-BEST Study (Benefit and efficacy in severely demented patients during treatment with memantine), Int $J$ Geriatr Psychiatry 14 (1999), 135-146.
[38] B. Winblad, H.J. Mobius and A. Stoffler, Glutamate receptors as a target for Alzheimer's disease - are clinical results supporting the hope? J Neural Transm 62 (2002), 217-225. 